\title{
There's a goat behind door number 3: from Monty Hall to medicine
}

\author{
David J. Friedman, ${ }^{1}$ Laurence A. Turka, ${ }^{1,2}$ and Simon C. Robson ${ }^{1}$
}

${ }^{1}$ Beth Israel Deaconess Medical Center and Harvard Medical School, Boston, Massachusetts, USA. 2 Immune Tolerance Network, Boston, Massachusetts, USA.

\begin{abstract}
In the 18th century, Thomas Bayes developed his eponymous theorem that teaches us that pretest probabilities can be altered by new information, such as when game show host Monty Hall revealed the goat behind one of the remaining doors in "Let's Make A Deal." Bayesian analysis is a key feature of many medical decisions. In this issue of the JCI, Lee and colleagues apply this concept to inflammatory bowel disease to identify gene expressionbased biomarkers of disease severity. Importantly, these biomarkers allowed patients to be stratified into two groups: those at high risk for disease recurrence or the need for immunosuppressive treatment escalation and those with a more benign disease course.
\end{abstract}

Across disciplines, matching individual patients with the best therapy is a task that has become increasingly challenging for physicians, as the number of options for treatment has expanded. In some areas, investigators have had considerable success in helping physicians meet this challenge. For example, the identification of gene expression signatures associated with certain cancers allows oncologists to tailor therapy in order to maximize efficacy, without introducing the unnecessary toxicity of medications that will likely prove ineffective (1). Genetic tests, such as analysis of IL28B polymorphisms, have use in predicting the outcome of hepatitis $\mathrm{C}$ management (2). These advances have dramatically changed the approach to treating disease in this handful of illustrative situations, allowing the most severe cases to be treated most aggressively or even with an entirely different set of agents. In the absence of such biomarkers, physicians are often forced to rely on a "step-up" approach, escalating treatment in the setting of initial failures and perhaps missing important windows for the introduction of the optimal treatment. Even beyond the issue of patient stratification, biomarkers of disease activity can play a critical role in determining who needs and, just as importantly, who does not need treatment. Given the potent side effects of immunosuppressive drugs, including opportunistic infec-

Conflict of interest: Laurence A. Turka declares financial interests in Novartis and Myriad Genetics.

Citation for this article: J Clin Invest. 2011; 121(10):3819-3821. doi:10.1172/JCI60003. tions, malignancy, and cardiovascular disease, these treatment decisions are a key issue in the care of patients with autoimmune diseases, such as inflammatory bowel disease (IBD).

\section{Genetic studies:}

\section{lessons and limitations}

IBD actually refers to a group of conditions, the most common of which are Crohn disease (CD) and ulcerative colitis (UC). IBD, and CD in particular, is a devastating and relapsing illness that is associated with excessive inflammation in the intestinal tract in genetically susceptible individuals $(3,4)$. IBD is noteworthy for aberrant innate and adaptive immune responses, with increasing evidence to support a role for $\mathrm{T}$ cell dysfunction in the regulation of the response to mucosal injury (5). In agreement with this, genome-wide association studies (GWAS) of IBD have linked disease susceptibility to polymorphisms in genes associated with IL-23 signaling in Th17 cells (including the IL23R, IL12B, JAK2, TYK2, and STAT3 genes; ref. 6), suggesting that dysregulation of such signaling pathways has a role in disease pathogenesis. In $\mathrm{CD}$, genetic variants (e.g., those in the NOD2 gene) suggestive of defective processing of intracellular bacteria, disordered autophagy, and perturbed innate immunity are also linked to disease susceptibility (reviewed in ref. 6 and see also ref. 4).

Given this degree of knowledge, IBD might seem to be among the best candidate diseases for molecular profiling. However, although knowledge of genetic susceptibility loci has illuminated much about disease pathogenesis, these markers have not provided any sort of information to help in the clinical stratification of patients to guide therapy. This is because, as with virtually all other autoimmune diseases, the IBD susceptibility loci identified through GWAS explain only a small proportion of the disease heritability and, more importantly, do not predict response to therapy or outcome $(7,8)$.

As genetic studies have failed to identify clinically useful biomarkers of IBD activity and response to therapy, there has been substantial interest in developing ways to obtain this information using other approaches. Unfortunately, studies indicate that a battery of other potential markers, such as cytokine levels, flow cytometry of immune cells, and immune cell activation assays, yield little practical advice for the clinician. Complicating the search for these actionable biomarkers is the multitude of discrete $T$ cell subsets and other immune cell types implicated in the disease process. This poses a particularly difficult challenge for those seeking to examine the newest "wave" of biomarkers - namely, gene expression signatures. Peripheral blood is obviously the easiest tissue to sample in individuals with IBD, yet it has not been clear in which cells investigators should look for a gene expression signature, and examination of whole peripheral blood mononuclear cells can be problematic because of a potentially low signal-to-noise ratio.

In this issue of the JCI, Lee et al. have made a promising advance toward identifying biomarkers of IBD activity and response to therapy and thereby toward helping identify those who should receive potent immunosuppressive therapies, such as anti-TNF modalities (9). This current report builds on previous work from the same research group in anti-neutrophil cytoplasmic antibody-associated vasculitis (AAV) and SLE (10) to identify $\mathrm{T}$ cell gene expression signatures with surprisingly strong associations to IBD course and therapeutic responsive- 

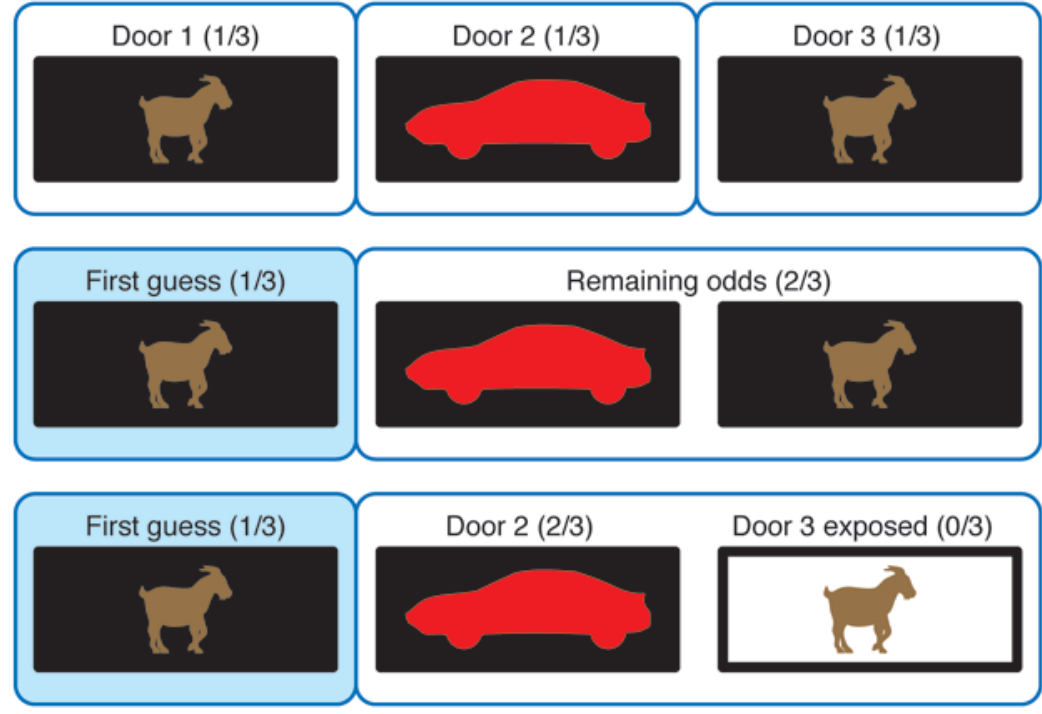

ness (9). These T cell gene expression signatures impressively separate patients with autoimmune disease at high risk for recurrence or treatment escalation from others with a more benign course.

\section{$\mathrm{CD}^{+} \mathrm{T}$ cells hold the answer}

A key element in addressing the considerable complexity of AAV and SLE was the adoption of a hypothesis-free, non-biased approach based on genome-wide profiling of multiple cell types (10). Instead of starting with a few particular cytokines or a pet cell type of interest, multiple immune cell types, including $\mathrm{CD}^{+} \mathrm{T}$ cells, $\mathrm{CD}^{+} \mathrm{T}$ cells, B cells, neutrophils, and monocytes, were individually profiled for gene expression signatures. In their earlier work (10), the authors discovered and validated a reproducible gene expression signature in $\mathrm{CD}^{+} \mathrm{T}$ cells (but not other immune cell types) that correlated strongly with disease response after initial treatment in AAV and then demonstrated that the same signature had similar prognostic value in SLE. In both AAV and SLE, poor prognosis was associated with increased expression of genes encoding proteins involved in signaling via the IL-7R and TCR pathways, signaling pathways implicated in $\mathrm{T}$ cell activation and the subsequent development of antigenspecific memory $\mathrm{T}$ cell populations. This may be indicative not of disease causation or initiation but rather of progression and responsiveness (typically seen in relapsing diseases). Importantly, these predictive signatures disappeared after initial therapy.

These signatures could be reduced to a manageable number of genes (i.e., three) and appeared to offer more insight to the clinician than traditional markers such as myeloperoxidase or C-reactive protein levels and other clinical indicators (10). In the current JCI manuscript, Lee et al. asked whether similar gene expression signatures existed for IBD (9), a less classical form of autoimmunity that appears to reflect disharmony between the immune system and gut flora rather than self antigens. The authors provide a compelling, affirmative answer. Again, it is somewhat of a surprise that in CD the authors found that it is the $\mathrm{CD}^{+} \mathrm{T}$ cells rather than the better-characterized $\mathrm{CD}^{+} \mathrm{T}$ cells linked to IBD that carry the useful signatures. Moreover, the $\mathrm{CD}^{+} \mathrm{T}$ cell gene expression pattern signifying poor prognosis in IBD mirrors the initial signatures described for AAV and SLE (10). Curiously, the informative genes had relatively little direct correlation with IBD genetic susceptibility alleles, such as NOD2 (11), and those encoding genes involved in Th17 cell signaling (12). The nature of the antigens that drive the $\mathrm{CD}^{+}$ $\mathrm{T}$ cell memory-type responses characterized by the gene expression signatures remains unknown at this time, as does any potential involvement of other cell types in the generation of such responses.

The $\mathrm{CD}^{+} \mathrm{T}$ cell gene expression signature identified by Lee and colleagues (9) impressively identifies IBD sufferers at high risk for disease recurrence or need for escalation of their immunosuppressive treatments, whereas traditional markers (such as anti-Saccharomyces cerevisiae status and clinical risk scores) offer essentially no diagnostic benefit. Lee and colleagues

\section{Figure 1}

The Monty Hall problem. In the game show "Let's Make a Deal," the contestant has to choose a door. The initial probability of the car being behind each of the doors is equal - one-third. After the contestant chooses door no. 1, the host reveals a goat behind door no. 3 and asks the contestant whether he/she wishes to switch their choice from door no. 1 to door no. 2. This is the correct option, because with this new information, the probability of the car being behind door no. 2 is now two-thirds. found that, for both CD and UC, essentially the same gene expression signatures (organized around genes involved in the signaling pathways triggered by IL-7, IL-2, and TCR ligation as well as elements of CD28 costimulation) aligned individuals at high and low risk for poor outcome (9). Using Bayesian analysis (as a knowledgeable contestant on "Let's Make a Deal" would have done; Figure 1), they showed that stratification using their biomarker signature greatly enhanced their ability to predict which patients required treatment escalation, suggesting that useful information to guide the course of IBD therapy may soon be at hand for clinicians. If these data can be validated in a prospective study, using this type of personalized approach to select patients prior to introduction of immunosuppressive therapies may become a clinical reality. It is a major advantage that the level of expression of just a few tagged genes can accurately represent immensely complicated signaling processes, and this is reason for optimism. In addition, the bimodal distribution of the prognostic gene expression signatures also suggests that sorting of patients into risk groups may be easier to accomplish than if it were a normally distributed variable. Nonetheless, any clinical test would need to incorporate cell-sorting techniques with those determining the levels of expression of these genes, adding an additional level of complexity to the assay.

The specifics of how the gene expression signatures identified by Lee and colleagues (9) may alter clinical care await carefully conducted trials, but in the future, guess- 
work may well be reduced in favor of meaningful, objective data. Just as importantly, these gene expression patterns may help refocus research efforts to understand the pathogenesis of IBD and the mechanisms of treatment resistance.

\section{Implications}

The fact that the gene expression signatures identified for AAV and SLE (10), which were very similar to those used by Lee and colleagues (9), could be used to divide normal controls into the same two groups that it did the patients with these various diseases indicates that these gene expression signatures stratify something beyond just a propensity to a particular disease. This suggests that these data indicate some preexisting set point for the inflammatory response that may be independent of any specific disease. We speculate that this non-Gaussian (bimodal) distribution of the population indicates that, in the past, natural selection favored the coordinated increase in gene expression for enhanced immunity and that this pattern of gene expression is now associated with more severe disease in IBD, SLE, and AAV vascular-type inflammation and perhaps other autoimmune diseases. Coordinating future studies with genome-wide surveys of genetic polymorphisms in larger populations may help identify the master regulator(s) of this particular gene expression signature; again, the bimodal distribution may hint that the number of these master regulators is smaller than expected.

In conclusion, if follow-up studies show that prestratifying IBD patients for different treatment strategies based on $\mathrm{CD}^{+} \mathrm{T}$ cell gene expression signatures can lead to improved outcomes, management of IBD will take a giant leap forward. Furthermore, the general approach of Lee et al. (9) may become a useful paradigm for managing potentially hazardous immunotherapies for this and other autoimmune diseases.

Address correspondence to: Laurence A. Turka, Transplant Institute, Beth Israel Deaconess Medical Center, 330 Brookline Avenue - E/CLS 607, Boston, Massachusetts 02215, USA. E-mail: 1turka@bidmc.harvard. edu. Or to: Simon C. Robson, Transplant Institute, Beth Israel Deaconess Medical Center, 330 Brookline Avenue - E/CLS 612, Boston, Massachusetts 02215, USA. E-mail: srobson@bidmc.harvard.edu.
1. Chang JC, et al. Gene expression profiling for the prediction of therapeutic response to docetaxel in patients with breast cancer. Lancet. 2003; 362(9381):362-369.

2. Tanaka Y, et al. Genome-wide association of IL28B with response to pegylated interferon-alpha and ribavirin therapy for chronic hepatitis C. Nat Genet. 2009;41(10):1105-1109.

3. Podolsky DK. Inflammatory bowel disease. N Engl J Med. 2002;347(6):417-429.

4. Khor B, Gardet A, Xavier RJ. Genetics and pathogenesis of inflammatory bowel disease. Nature. 2011; 474(7351):307-317

5. Strober W, Fuss I, Mannon P. The fundamental basis of inflammatory bowel disease. J Clin Invest. 2007; 117(3):514-521

6. Lees CW, Barrett JC, Parkes M, Satsangi J. New IBD genetics: common pathways with other diseases [published online ahead of print February 7, 2011]. Gut. doi:gut.2009.199679.

7. Lettre G, Rioux JD. Autoimmune diseases: insights from genome-wide association studies. Hum Mol Genet. 2008;17(R2):R116-R121.

8. Baranzini SE. The genetics of autoimmune diseases: a networked perspective. Curr Opin Immunol. 2009;21(6):596-605.

9. Lee JC, et al. Gene expression profiling of $\mathrm{CD}^{+}$ $\mathrm{T}$ cells predicts prognosis in patients with Crohn disease and ulcerative colitis. J Clin Invest. 2011; 121(10):4170-4179.

10. McKinney EF, et al. A CD8+ T cell transcription signature predicts prognosis in autoimmune disease. Nat Med. 2010;16(1):586-591.

11. Bonen DK, Cho JH. The genetics of inflammatory bowel disease. Gastroenterology. 2003;124(2):521-536.

12. Stockinger B, Veldhoen M, Martin B. Th17 T cells: linking innate and adaptive immunity. Semin Immunol. 2007;19(6):353-361.

\section{Lessons in human biology from a monogenic pancreatic $\beta$ cell disease}

\section{Benjamin Glaser}

Endocrinology and Metabolism Service, Internal Medicine Department, Hadassah-Hebrew University Medical Center, Jerusalem, Israel.

\begin{abstract}
Deciphering the complexities of human $\beta$ cell physiology is critical to our understanding of the pathophysiology behind both type 1 and type 2 diabetes. One way to do this is to study individuals with congenital hyperinsulinism (CHI), a rare genetic disease characterized by dysregulation of insulin secretion resulting in hypoglycemia. In this issue of the JCI, Henquin et al. report in vitro studies of pancreatic tissue obtained from CHI patients during therapeutic pancreatectomy that have yielded exciting new insights into human $\beta$ cell physiology. The data validate and extend observations made in model organisms.
\end{abstract}

$\beta$ Cell dysfunction lies at the center of both major forms of diabetes, the incidence of which is increasing at an alarming rate in both the developed and the developing world (1). In type 1 diabetes mellitus

Conflict of interest: The author has declared that no conflict of interest exists.

Citation for this article: J Clin Invest. 2011; 121(10):3821-3825. doi:10.1172/JCI60002.
(T1DM), autoimmune $\beta$ cell destruction results in complete insulin deficiency, whereas in type 2 diabetes mellitus (T2DM), subtle defects in $\beta$ cell functional mass result in progressive disease. Studies using animal models and cell lines from various sources have yielded invaluable information that has allowed us to better understand the processes responsible for $\beta$ cell function, dysfunction, replication, and survival.
However, since the model systems may not always accurately reflect in vivo human physiology, validation studies in humans are crucial. The crunch comes, however, in that performing such studies is at best difficult and often impossible. Genetic manipulation in vivo, a hugely powerful tool in animal-based research, is obviously impossible in humans, and in vitro genetic manipulations of primary human tissues are difficult and in themselves fraught with pitfalls.

One way to overcome some of these problems is to identify and study individuals with naturally occurring genetic mutations. These cases are rare and often difficult to study, and the results may be difficult to interpret. With animal models, experiments can be performed in genetically identical animals under controlled conditions. In human studies, however, each patient typi- 